\title{
Determinação da força de arrasto e da velocidade terminal de frutos de café pela técnica de elementos finitos

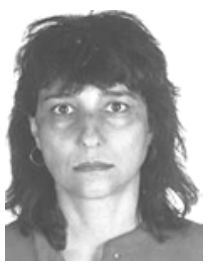 \\ Sandra M. Couto ${ }^{1}$, Ana E. O. dos Santos ${ }^{2}$, Stella M. J. Vieira ${ }^{3} \&$ Danilo J. P. da Silva ${ }^{4}$ \\ 1 DEA/UFV. CEP 36571-000, Viçosa, MG. Fone: (31) 3899-1927. E-mail: scouto@ufv.br (Foto) \\ 2 UFV. E-mail: anaelisaos@bol.com.br \\ ${ }^{3}$ UFV. E-mail: smjvieira@bol.com.br \\ ${ }^{4}$ UFV. E-mail: danilojpsilva@yahoo.com.br
}

Protocolo $129-2 / 9 / 2002$ - Aprovado em 16/2/2004

\begin{abstract}
Resumo: Este trabalho apresenta um método alternativo para a determinação de velocidades terminais e de forças de arrasto em frutos de café, ao se variar o teor de umidade do produto e sua configuração geométrica (esférica e elíptica). Especificamente, investigou-se a aplicabilidade da técnica de elementos finitos para a obtenção de: (a) forças de arrasto no produto, usando-se valores experimentais das dimensões do fruto e de sua velocidade terminal e (b) velocidades terminais do produto, empregando-se valores experimentais das dimensões do fruto e de seu peso. A técnica dos elementos finitos mostrou-se ferramenta eficiente para simular velocidades terminais e forças de arrasto em frutos de café submetidos a um fluxo de ar. Independente do teor de umidade dos frutos, os valores simulados para a velocidade terminal e para força de arrasto no produto considerado elíptico são, estatisticamente, iguais àqueles determinados experimentalmente. Os erros relativos médios, envolvidos no valores simulados para a velocidade terminal do produto, em cada um dos teores de umidade, não foram superiores a 7,5 e 13,6\% para o produto considerado elíptico e esférico, respectivamente.
\end{abstract}

Palavras-chave: configuração geométrica, força de arrasto, Coffea arabica L.

\section{Determination of drag force and terminal velocity of coffee fruits using finite element technique}

\begin{abstract}
This work presents an alternative method for terminal speed and drag force determinations for coffee fruits with different moisture contents and geometric configurations (spherical and elliptic). Specifically, the use of the finite element technique was investigated for obtaining: (a) product drag forces using experimental values of the fruit dimensions and its terminal velocity and (b) product terminal velocity using experimental values of fruit dimensions and weight. The use of finite element technique was shown to be an efficient tool to simulate terminal velocity and drag force on coffee fruits submitted to airflow. Independent of the fruit moisture content, simulated and experimental values for the terminal velocity and drag force did not differ statistically when the product was considered elliptic. For each product moisture content, the mean relative errors involved in the simulated values of terminal velocity were not superior to 7.5 and $13.6 \%$ for the fruits considered elliptic and spherical, respectively.
\end{abstract}

Key words: geometric configuration, drag force, Coffea arabica L.

\section{INTRODUÇÃO}

O conhecimento da velocidade terminal de um produto e da força de arrasto em partículas colocadas em fluidos em movimento é sempre necessário em muitas aplicações em engenharia, tais como transporte pneumático e hidráulico de um produto e processos de separação e classificação de materiais. Determinações experimentais da velocidade terminal de vários tipos de grãos e sementes foram realizadas no passado e, atualmente, ainda continuam sendo o objetivo de vários pesquisadores (por exemplo: Carman, 1996; Singh \& Goswami, 1996; Suthar \& Das, 1996; Allen \& Watts, 1997; Gupta \& Das, 1997; Nimkar \& Chattopadhyay, 2001). Devido a importância da velocidade terminal e da força de arrasto, um grande número de 
pesquisas tem sido realizado objetivando o desenvolvimento de métodos de predição precisos para estimar estas grandezas; entretanto, a maioria dessas pesquisas é direcionada para materiais não agrícolas.

De acordo com Fox \& McDonald (1998), a superfície de um corpo imerso em um fluido em movimento, fica submetida a uma força dinâmica, resultante de forças de pressão (normal à superfície) e de cisalhamento (tangente à superfície). Tensões cisalhantes devido à viscosidade e gradientes de velocidade na superfície do corpo causam forças tangenciais à superfície enquanto variações nas intensidades de pressão ao longo da superfície, devido aos efeitos dinâmicos, resultam em forças normais à superfície do corpo. A componente da força total exercida pelo fluido no sólido, na direção do escoamento é denominada arrasto.

A força de arrasto atuante em um corpo é, portanto, composta de duas parcelas: uma devida ao arrasto de atrito e a outra ao arrasto de pressão. A contribuição relativa de cada uma varia com o regime do escoamento e com as condições da superfície do corpo. $\mathrm{O}$ arrasto de pressão depende, basicamente, da forma do corpo e pode ser reduzido a um mínimo como, por exemplo, em um elipsóide de revolução exposto a um escoamento paralelo ao eixo de simetria do corpo. O arrasto de atrito não depende significativamente da forma do corpo; entretanto, é influenciado pelas características da superfície,Tabak \& Wolf(1998), Dong et al.(2001).

Os arrastos de pressão e atrito são simultaneamente de grande valor apenas em poucas situações. Para objetos que não apresentam qualquer sustentação, o arrasto de pressão pode ser considerado como o arrasto total, Giles (1980). Segundo Fox \& McDonald (1998), no caso de escoamento sobre uma esfera lisa, tanto o arrasto de atrito quanto o de pressão contribuem para o arrasto total. Para números de Reynolds, Re, muito baixos $(\operatorname{Re} \leq 1)$, o arrasto é predominantemente devido ao atrito. À medida que o número de Reynolds aumenta até cerca de 1000, o arrasto é uma combinação das parcelas devidas ao atrito e à pressão. A contribuição relativa do arrasto de atrito cai com o aumento do número de Reynolds; para $\mathrm{Re} \approx 1000$, o arrasto de atrito é aproximadamente $5 \%$ do arrasto total.

A força de arrasto (F) em um corpo imerso em um fluido em movimento é comumente expressa como $\mathrm{F}=\frac{1}{2} \mathrm{CA} \rho \mathrm{V}^{2}$, em que $\mathrm{C}$ é o coeficiente de arrasto (adimensional); $\mathrm{A}$ é a área característica $\left(\mathrm{m}^{2}\right)$; é a densidade do fluido $\left(\mathrm{kg} \mathrm{m}^{-3}\right)$ e $\mathrm{V}$ é a velocidade uniforme do fluido $\left(\mathrm{m} \mathrm{s}^{-1}\right)$. $\mathrm{O}$ coeficiente de arrasto inclui os arrastos de pressão e de atrito.

A determinação da velocidade terminal de corpos, baseandose em expressões que relacionam o coeficiente de arrasto e o número de Reynolds, tem sido extensivamente investigada por vários pesquisadores nos últimos noventa anos. Nguyen et al. (1997) relatam que, atualmente, existem pelo menos 35 equações na literatura que expressam o coeficiente de arrasto (C) sobre uma partícula em função do número de Reynolds. A determinação da velocidade terminal através de quaisquer dessas equações propostas para "C versus Re", requer um procedimento de tentativa e erro, visto que a velocidade se encontra presente nas duas variáveis e a relação entre "C e Re" é altamente não linear.
A velocidade terminal e, conseqüentemente, o coeficiente de arrasto de uma partícula são por demais influenciados pela sua forma, tamanho e orientação, pela viscosidade do meio e pelas densidades da partícula e do fluido. Dos modelos semiempíricos desenvolvidos para predizer forças de arrasto em corpos submersos em um fluido, grande parte considera as partículas tendo uma forma esférica, apesar das partículas envolvidas em processos de engenharia não terem, na grande maioria, esta forma. Atualmente, é possível se estimar forças de arrasto ou velocidades terminais de uma partícula esférica sob a maioria das condições de interesse prático em um meio newtoniano incompressível. Entretanto, quando se trata de partículas não esféricas, a falta de padrões para a medição da sua forma, seu tamanho e orientação durante a flutuação, de acordo com Chhabra et al. (1999), tem sido um dos grandes obstáculos para obtenção de modelos adequados para predizer forças de arrasto e no desenvolvimento de correlações aplicáveis universalmente.

Arsenijevic et al. (1999) desenvolveram um método para a determinação da velocidade terminal de partículas não esféricas, no qual usaram dados de queda de pressão em um leito recheado do produto para a determinação indireta do fator de forma para a partícula. Segundo os autores, os outros métodos existentes necessitam do conhecimento da forma da partícula, parâmetro de difícil obtenção para materiais reais.

Chhabra et al. (1999) investigaram a eficácia de correlações mais comumente usadas para estimar o coeficiente de arrasto de partículas não esféricas em fluidos viscosos incompressíveis e observaram que a influência da forma da partícula sobre o coeficiente de arrasto aumenta com o número de Reynolds e, ainda, que melhores estimativas desta grandeza poderiam ser obtidas somente se parâmetros ligados à forma da partícula fossem adicionados às correlações.

Teoricamente, a velocidade terminal de um partícula e a força de arrasto podem ser determinadas por meio de uma análise do escoamento de um fluido sobre uma superfície sólida (partícula). Para condições isotérmicas, isto envolve a resolução simultânea das equações de conservação de massa e de conservação do momentum. Na maioria dos casos, soluções analíticas para este tipo de problema não podem ser viabilizadas e se faz necessário a adoção de algum método numérico para a obtenção de soluções.

O método dos elementos finitos, segundo Moaveni (1999), é um procedimento numérico que pode ser usado para a obtenção de soluções para uma extensa série de problemas relacionados com engenharia, incluindo escoamento de fluidos. A vantagem deste método sobre os outros reside no fato de ser aplicável a objetos de formas irregulares, compostos de diferentes materiais e tendo condições de contorno mistas. Sendo um método numérico, as soluções, obtidas por esta técnica, aproximam as soluções exatas somente em pontos discretos do sistema, denominados nós. Para a aplicação do método o sistema é, inicialmente, discretizado em pequenas sub-regiões, denominadas elementos, conectadas através de pontos (nós). $\mathrm{O}$ método usa formulações integrais para criar um sistema de equações algébricas. Uma função contínua aproximada é usada para representar a solução para cada elemento. A solução completa é gerada pela conexão das soluções individuais. 
Torna-se evidente, diante do exposto, a necessidade de métodos alternativos mais eficazes para a determinação de velocidades terminais e de forças de arrasto em partículas não esféricas, tais como a maioria dos produtos agrícolas. Neste sentido, o trabalho teve, por objetivo, o desenvolvimento de uma metodologia para a determinação de propriedades aerodinâmicas de frutos de café, ao se variar o teor de umidade do produto e sua configuração geométrica (esférica e elíptica). Especificamente, investigou-se a aplicabilidade da técnica de elementos finitos para a obtenção de: (a) forças de arrasto em frutos de café, usando-se valores experimentais das dimensões do fruto e de sua velocidade terminal e (b) velocidades terminais do produto através de valores experimentais das dimensões do fruto e de seu peso.

\section{MATERIAL E MÉTODOS}

\section{Predição da força de arrasto nos frutos de café}

Em geral, a superfície de um corpo imerso em um fluido, em cada ponto, faz um ângulo com a direção do escoamento e, neste caso, o arrasto total em um elemento de área da superfície, dA, é a soma das componentes, na direção do escoamento, da força cisalhante ( $\tau \mathrm{dA})$ e da força de pressão do fluido (PdA) como esquematizado na Figura 1. Considerando-se o elemento de área, dA, inclinado de um ângulo de $\left(90^{\circ}\right.$ - á ) em relação à direção do escoamento, o arrasto devido ao cisalhamento (arrasto de atrito) é ô sen á dA e aquele devido à pressão (arrasto de forma ou de pressão) é igual a $\mathrm{P} \cos$ ô $\mathrm{dA}$. O arrasto total sobre um corpo é a soma das integrais dessas duas quantidades sobre toda a superfície do corpo em contato com o fluido.

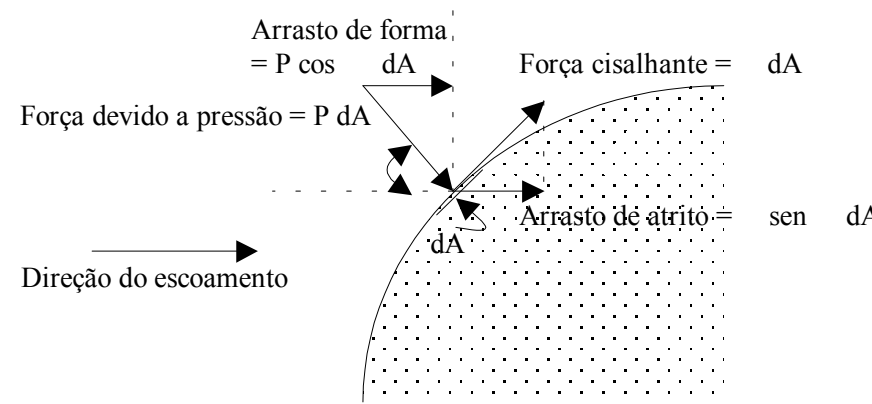

Figura 1. Arrasto de atrito e de forma em um corpo imerso em um fluido

A metodologia apresentada neste trabalho para a determinação de forças de arrasto em frutos de café considera:

- escoamento de ar em um duto circular (raio $=0,046 \mathrm{~m} \mathrm{e}$ comprimento $=1,0 \mathrm{~m}$ ) contendo, no seu interior, um fruto de café;

- fruto posicionado no eixo longitudinal central do duto e na metade do seu comprimento;

- velocidade do ar no duto equivalente à velocidade terminal do produto. Portanto, o fruto permanece estacionário no interior do duto, durante o escoamento de ar;

- sistema investigado corresponde à metade da seção longitudinal do duto, como esquematizado na Figura 2A, com simetria em relação ao eixo longitudinal central do duto $(\mathrm{X})$, paralelo à direção do fluxo de ar;
- efeito tridimensional: obtido através da rotação do sistema em torno do eixo de simetria $(\mathrm{X})$ durante as análises;

- forma geométrica do fruto de café no interior do duto: esférica ou elíptica.

- dimensões características do fruto: A e B, Figura 2A. No caso do fruto elíptico: $A=a / 2$ e $B=(c+b) / 4$, Figura $2 B$. Para fruto esférico: $R E=A=B=(a+b+c) / 4$, em que $R E$ é o raio equivalente esférico do fruto;

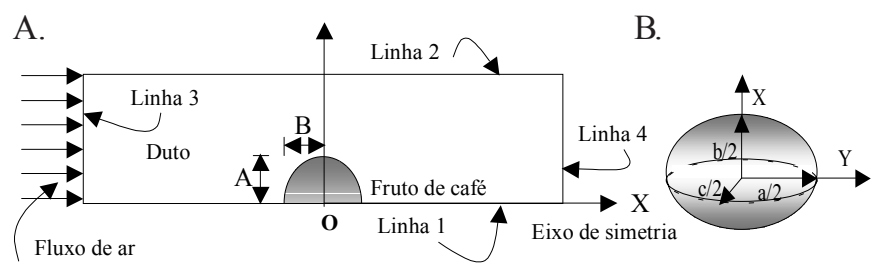

Figura 2. (A) Esquema do sistema submetido a análise: metade da seção longitudinal do duto e (B) dimensões principais do fruto de café

- desprezível a contribuição do arrasto de atrito para o arrasto total nos frutos, ou seja, nas análises considerou-se apenas o arrasto de pressão.

Para a determinação da força de arrasto no produto, independente da forma assumida para o fruto, na sua superfície (projeção de sua metade no plano XY) foram especificados trinta e sete pontos (defasados de cinco graus), como pode ser visualizado na Figura $3 \mathrm{~A}$.

Os valores de pressão total nos 37 pontos (nós) foram considerados previamente conhecidos (provenientes de análise do escoamento de ar no duto pela técnica de elementos finitos). Qualquer um dos pontos representados na Figura 3A, por exemplo: o denominado "N", pode ser localizado em relação à origem do sistema de referência, pelo ângulo " $\theta$ " ou pelas suas coordenadas " $\mathrm{X}_{\mathrm{N}}$ " e " $\mathrm{Y}_{\mathrm{N}}$ ".

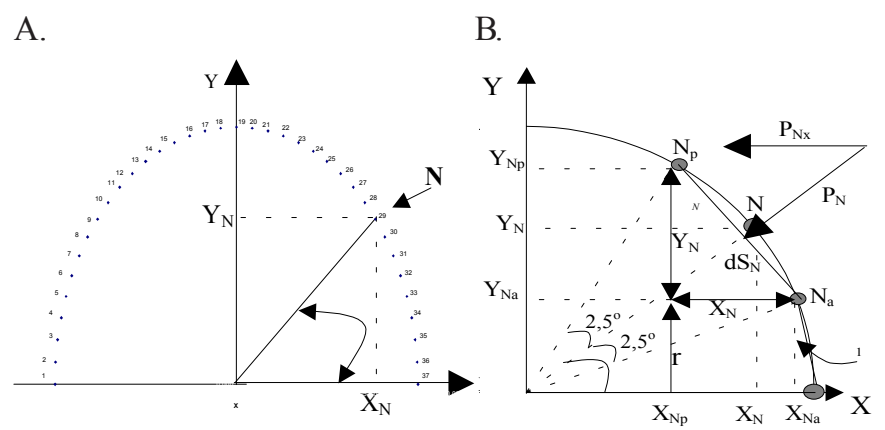

C.
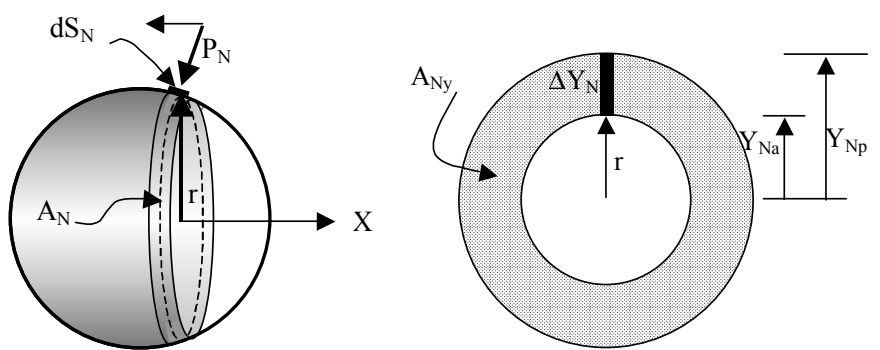

Figura 3. Esquema usado para a determinação das forças de arrasto (A) Nós na superfície do fruto (B) Aproximações consideradas - Nó "N" (C) área atuada pela pressão $\mathrm{P}_{\mathrm{N}}$ 
Conforme mostrado na Figura 3B, em torno de cada um dos 37 nós foram construídos pontos intermediários, denominados $\mathrm{Na}$ (anterior ao nó $\mathrm{N}$, com coordenadas $\mathrm{X}_{\mathrm{Na}}$ e $\mathrm{Y}_{\mathrm{Na}}$ ) e $\mathrm{Np}$ (posterior ao nó $\mathrm{N}$, com coordenadas $\mathrm{X}_{\mathrm{Np}}$ e $\mathrm{Y}_{\mathrm{Np}}$ ), cada um defasado de 2,5 graus do nó $\mathrm{N}$. O segmento de arco entre os dois pontos intermediários, vizinhos de "N", foi aproximado por um segmento de reta de comprimento $\mathrm{dS}_{\mathrm{N}}$, no qual a pressão correspondente ao nó "N" passou a atuar.

As equações usadas para a obtenção da força de arrasto nos frutos, considerados esferas e elipses, são apresentadas na Tabela 1, bem como as expressões e aproximações utilizadas para as grandezas envolvidas nas determinações. Os parâmetros principais presentes nas equações podem ser visualizados na Figura 3B e C.

Tabela 1. Equações, expressões e aproximações usadas para a obtenção da força de arrasto no fruto considerados esferas e elipses.

\begin{tabular}{|c|c|c|}
\hline & Esfera & Elipse \\
\hline $\begin{array}{l}\text { Equação da } \\
\text { Superfície } \\
\text { do fruto }\end{array}$ & $X^{2}+Y^{2}=(R E)^{2}$ & $\left(\frac{X}{B}\right)^{2}+\left(\frac{Y}{A}\right)^{2}=1$ \\
\hline $\begin{array}{l}\text { Grandezas } \\
\text { de referência }\end{array}$ & $\theta, d \theta, R_{E}, P_{N}$ & $\begin{array}{c}\alpha \\
X_{N a}, Y_{N a}, X_{N p}, Y_{N p}, P_{N}\end{array}$ \\
\hline $\begin{array}{r}\text { Força de } \\
\operatorname{arrasto}(F)\end{array}$ & $F=\sum_{N=1}^{H} d F_{N}=\sum_{N=1}^{H} P_{N x} d A_{N y}$ & $F=\sum_{N=1}^{H} d F_{N}=\sum_{N=1}^{H} P_{N x} d A_{N y}$ \\
\hline$P_{N x}$ & $P_{N} \cos \theta$ & $P_{N} \cos \alpha_{N}$ \\
\hline$d A_{N y}$ & $d A_{N} \cos \theta$ & $\pi\left(\Delta Y_{N}\right)\left(Y_{N p}+Y_{N a}\right)$ \\
\hline $\cos \alpha_{N}$ & - & $\frac{\Delta Y_{N}}{d S_{N}}=\frac{\Delta Y_{N}}{\sqrt{\left(\Delta Y_{N}\right)^{2}+\left(\Delta X_{N}\right)^{2}}}$ \\
\hline$\Delta Y_{N}$ & - & $Y_{N p}-Y_{N a}$ \\
\hline$\Delta X_{N}$ & - & $X_{N p}-X_{N a}$ \\
\hline$d S_{N}$ & $R_{E} d \theta$ & $\sqrt{\left(\Delta Y_{N}\right)^{2}+\left(\Delta X_{N}\right)^{2}}$ \\
\hline$d A_{N}$ & $2 \pi r R_{E} d \theta$ & \\
\hline$r$ & $R_{E} \operatorname{sen} \theta$ & \\
\hline$d F_{N}$ & $2 \pi P_{N} R_{E}^{2} d \theta \cos ^{2} \theta \operatorname{sen} \theta$ & $\frac{\mathrm{P}_{\mathrm{N}} \pi\left(\Delta \mathrm{Y}_{\mathrm{N}}\right)^{2}\left(Y_{\mathrm{N}_{p}}+Y_{\mathrm{N} a}\right)}{\sqrt{\left(\Delta \mathrm{Y}_{\mathrm{N}}\right)^{2}+\left(\Delta X_{N}\right)^{2}}}$ \\
\hline
\end{tabular}

em que:

H - número total de nós para os quais a pressão é conhecida

$d S_{N}$ - magnitude do segmento de re

$F$ - força de arrasto total sobre o fruto

$F_{N}$ - força de arrasto sobre o elemento $\mathrm{N}$

$P_{N x}$ - componente, na direção $\mathrm{X}$, da pressão atuante no elemento $\mathrm{N}$

$\mathrm{R}_{\mathrm{E}}$ - raio equivalente da esfera

$A_{N y}$ - componente de área do elemento $\mathrm{N}$, perpendicular a $\mathrm{P}_{\mathrm{N} x}$

$A_{N}$ - área da superfície do fruto atuada pela pressão $\mathrm{P}_{\mathrm{N}}$

$r \quad$ - dimensão que delimita a área de cada elemento (Figura $\mathrm{x}$ )

$F_{N i}$ - força de arrasto nos elementos sem contato com o eixo $\mathrm{X}$
$F_{N e}$ - força de arrasto nos elementos em contato com o eixo $\mathrm{X}$

As forças de arrasto sobre as áreas superficiais correspondentes aos nós pertencentes ao primeiro quadrante da esfera ou da elipse, foram consideradas negativas enquanto aquelas referentes aos nós do segundo quadrante, foram consideradas positivas. No caso do fruto elíptico, para nós da superfície em contato com o eixo $\mathrm{X}$, os valores de $\mathrm{X}_{\mathrm{Na}}$ e $\mathrm{Y}_{\mathrm{Na}}$, concernente ao nó da direita, e $\mathrm{X}_{\mathrm{Np}}$ e $\mathrm{Y}_{\mathrm{Np}}$, correspondente ao nó da esquerda, foram considerados iguais a zero na equação correspondente na Tabela 1 .

\section{Escoamento de ar no duto - Análise por Elementos Finitos}

Valores de pressão total nos trinta e sete nós, Figura 3(A), especificados previamente na superfície do fruto submetido a um fluxo de ar, foram obtidos através de uma análise de escoamento de fluido usando-se a técnica de elementos finitos. O problema de escoamento (isotérmico, incompressível e em regime permanente) de fluidos é definido pelas leis de conservação de massa, Eq. 1, e de momentum, eqs. 2,3 e 4 .

$$
\text { Conservação de massa: } \frac{\partial V_{x}}{\partial x}+\frac{\partial V_{y}}{\partial y}+\frac{\partial V_{z}}{\partial z}=0
$$

em que:

$\mathrm{V}_{\mathrm{x}}, \mathrm{V}_{\mathrm{y}}$ e $\mathrm{V}_{\mathrm{z}}=$ componentes da velocidade nas direções $\mathrm{x}, \mathrm{y}$ e $z$, respectivamente.

Equações de momentum:

$$
\begin{gathered}
\rho\left(\frac{\partial\left(V_{x} V_{x}\right)}{\partial x}+\frac{\partial\left(V_{y} V_{x}\right)}{\partial y}+\frac{\partial\left(V_{z} V_{x}\right)}{\partial z}\right)= \\
\rho g_{x}-\frac{\partial P}{\partial x}+\mu_{e}\left(\frac{\partial^{2} V_{x}}{\partial x^{2}}+\frac{\partial^{2} V_{x}}{\partial y^{2}}+\frac{\partial^{2} V_{x}}{\partial z^{2}}\right) \\
\rho\left(\frac{\partial\left(V_{x} V_{y}\right)}{\partial x}+\frac{\partial\left(V_{y} V_{y}\right)}{\partial y}+\frac{\partial\left(V_{z} V_{y}\right)}{\partial z}\right)= \\
\rho g_{y}-\frac{\partial P}{\partial y}+\mu_{e}\left(\frac{\partial^{2} V_{y}}{\partial x^{2}}+\frac{\partial^{2} V_{y}}{\partial y^{2}}+\frac{\partial^{2} V_{y}}{\partial z^{2}}\right) \\
\rho\left(\frac{\partial\left(V_{x} V_{z}\right)}{\partial x}+\frac{\partial\left(V_{y} V_{z}\right)}{\partial y}+\frac{\partial\left(V_{z} V_{z}\right)}{\partial z}\right)= \\
\rho g_{z}-\frac{\partial P}{\partial z}+\mu_{e}\left(\frac{\partial^{2} V_{z}}{\partial x^{2}}+\frac{\partial^{2} V_{z}}{\partial y^{2}}+\frac{\partial^{2} V_{z}}{\partial z^{2}}\right)
\end{gathered}
$$

em que:

$\mathrm{P}$ - pressão do fluido

gx, gy e gz - componentes da aceleração da gravidade nas direções das coordenadas espaciais $\mathrm{x}, \mathrm{y}$ e $\mathrm{z}$, respectivamente

$$
\begin{aligned}
& \mu_{e} \text { - viscosidade efetiva } \\
& \rho \text { - densidade do fluido }
\end{aligned}
$$


Para a análise do problema de escoamento usou-se um programa computacional: ANSYS (versão 5.3), especialista em soluções usando a técnica de elementos finitos. Este programa possui uma opção denominada FLOTRAN CFD (Computational Fluid Dynamics), dirigida para a resolução de problemas em escoamento de fluidos, utilizada neste trabalho. As equações diferenciais parciais que expressam as leis de conservação de massa e de momentum, são discretizadas com uma técnica baseada em elementos finitos. O processo de discretização consiste em derivar matrizes para os elementos, que serão acopladas em uma matriz global. O método de Garlekin dos resíduos ponderados é usado na obtenção das matrizes para os elementos. As matrizes dos elementos após serem formadas, são acopladas e o sistema de equações resultante é resolvido para cada grau de liberdade (velocidade e pressão), separadamente. O problema de escoamento é não linear e as equações governantes encontram-se acopladas.

FLOTRAN CFD disponibiliza dois tipos de método para a solução de sistemas de equações; o primeiro é um método rápido e aproximado: TDMA (Tri-Diagonal Matrix Algorithm) e o segundo abrange métodos semidiretos: métodos direcionais conjugados. Os dois tipos são iterativos. TDMA é usado para se obter a solução aproximada e os métodos direcionais conjugados quando soluções "exatas" são requeridas. Em certas situações, soluções exatas para as equações devem ser obtidas, enquanto em outras, soluções aproximadas são adequadas. $\mathrm{Na}$ solução do algoritmo seqüencial neste trabalho, o método TDMA (Tri-Diagonal Matrix Algorithm) foi usado para a solução das equações de momentum e o método direcional conjugado, gradiente conjugado precondicionado, foi usado na solução da equação de pressão.

Os passos básicos envolvidos na resolução do problema em que foi usada a técnica dos elementos finitos, foram: (1) discretização do sistema - divisão do domínio em elementos e nós; (2) consideração de uma função contínua aproximada para representar a solução de um elemento; (3) desenvolvimento de equações para um elemento; (4) conexão das soluções dos elementos para se obter a solução completa do problema (construção da matriz de rigidez global); (5) aplicação das condições iniciais e de contorno no sistema, bem como as forças que nele atuam; (6) resolução simultânea de um conjunto de equações algébricas para obter as soluções nos nós. Como resultado das análises, foram obtidas velocidades e pressões em cada um dos nós dos elementos que compunham o sistema.

Para a análise, o sistema (Figura 2A) foi dividido em elementos bidimensionais do tipo FLUID141, quadrilateral com quatro nós (pertencente à biblioteca do ANSYS). Este tipo de elemento permite regiões fluidas e sólidas no sistema. Elementos com propriedades relativas ao fluido foram usados na região onde ocorria o escoamento de ar e outros, com propriedades de sólido, foram usados para a região representando o fruto de café.

Os parâmetros de entrada para a execução do programa foram: dimensões do sistema relativas ao duto de escoamento; propriedades e características físicas do produto (dimensões relevantes à forma assumida pelo fruto; peso e densidade), propriedades do ar (densidade e viscosidade); velocidade terminal do produto e condições de contorno para o sistema.
Para se estimar a força de arrasto em frutos de café submetidos a um fluxo de ar, as seguintes propriedades e características do produto foram determinadas experimentalmente: as três dimensões principais do fruto ( $a, b$ e c, Figura 2B), massa e volume. As dimensões foram medidas por meio de um paquímetro $( \pm 0,001 \mathrm{~cm})$, a massa do fruto foi obtida em uma balança semi-analítica $\left(10^{-3} \mathrm{~g}\right)$ e o volume do produto foi determinado pelo método de complementação de volume seguindo a metodologia descrita por Moreira et al. (1984/85). A densidade do produto foi calculada pela razão entre a massa do fruto e seu volume.

As dimensões principais foram também empregadas para a obtenção da esfericidade, $\phi$, do produto,

$$
\left.\phi \phi=\frac{(a \cdot b \cdot c)^{1 / 3}}{a}\right]
$$

Determinou-se experimentalmente a velocidade terminal dos frutos de café utilizando-se um dispositivo constituído de um ventilador centrífugo acoplado a um tubo de acrílico transparente (0,092 $\mathrm{m}$ de diâmetro e 2,0 $\mathrm{m}$ de comprimento), posicionado na vertical. Após ser acomodado no centro de um compartimento telado posicionado a $1,0 \mathrm{~m}$ da extremidade inferior do tubo de acrílico, o fluxo de café era submetido a um fluxo de ar, cuja intensidade era aumentada até que ocorresse o início de flutuação do produto. A velocidade do ar no local de flutuação do fruto, medida por meio de um anemômetro de fio quente, foi considerada como a velocidade terminal do produto. Obtiveramse as velocidades terminais de frutos cujas dimensões, massas e volumes, foram previamente determinados.

Nos experimentos para a determinação das propriedades e características físicas do produto e sua velocidade terminal, foram usados frutos de café da variedade Catuaí, no estádio de maturação correspondente à coloração "cereja", com teores de umidade iguais a 67,$5 ; 46,9$ e $18,7 \%$ b.u. Para cada teor de umidade do produto foram investigados dez frutos.

As condições impostas aos contornos do sistema (referentes às linhas 1, 2, 3 e 4, esquematizadas na Figura 2A) envolveram, respectivamente, valores nulos das componentes de velocidade do ar nas direções y e z no eixo de simetria do sistema (X); valores nulos das componentes da velocidade do ar nas paredes do duto (condição de não deslizamento); velocidade homogênea do ar, igual à velocidade terminal do produto, na entrada do duto e pressão nula (referência) na saída do duto.

\section{Predição da velocidade terminal do produto}

Obtiveram-se valores para as velocidades terminais dos frutos, através do processo de otimização. Para tal, modificações foram feitas no programa fonte (elementos finitos) usado para a determinação das forças de arrasto no fruto de café, considerado esfera e elipse. Uma rotina de otimização foi introduzida no programa e os parâmetros de entrada passaram a ser: peso real do fruto, raio equivalente esférico (fruto esférico) ou dimensões "A" e "B" (fruto elíptico), os quais envolvem menores incertezas em seus valores que as velocidades terminais do produto obtidas experimentalmente. O objetivo da otimização foi determinar um valor para a velocidade terminal do produto, tal que o erro relativo 
entre a força de arrasto simulada usando esta velocidade e o peso real do fruto, fosse menor ou igual $0,1 \%$.

\section{RESULTADOS E DISCUSSÃO}

Os valores experimentais das dimensões principais volume, densidade real e esfericidade de cada um dos dez frutos de café, usados nas simulações nos teores de umidade (TU) de 67,8; 46,9 e 18,7\% b.u., são apresentados na Tabela 2.

Os valores de densidade do produto, apresentados na Tabela 2, de peso e de velocidade terminal (VT) expostos na Tabela 3, foram usados como entrada no programa computacional para estimar a força de arrasto no produto, usando-se a técnica de elementos finitos. Nas simulações, os valores de densidade e

Tabela 2. Propriedades e características físicas dos frutos de café

\begin{tabular}{|c|c|c|c|c|c|c|c|}
\hline \multirow{2}{*}{$\begin{array}{l}\text { TU* } \\
\% \text { b.u. }\end{array}$} & \multirow[b]{2}{*}{ Fruto } & \multicolumn{3}{|c|}{ Dimensão $\left(10^{-2} \mathrm{~m}\right)$} & \multirow{2}{*}{$\begin{array}{l}\text { Volume } \\
\left(10^{-6} \mathrm{~m}^{3}\right)\end{array}$} & \multirow{2}{*}{$\begin{array}{c}\text { Dens. } \\
\left(\mathrm{kg} \mathrm{m}^{-3}\right)\end{array}$} & \multirow[b]{2}{*}{ Esf. } \\
\hline & & $\mathrm{a}$ & $\mathrm{b}$ & $\mathrm{c}$ & & & \\
\hline \multirow[t]{12}{*}{67,8} & 1 & 1,56 & 1,42 & 1,40 & 1,6238 & 1231,7 & 0,93 \\
\hline & 2 & 1,49 & 1,36 & 1,24 & 1,3157 & 1276,9 & 0,91 \\
\hline & 3 & 1,40 & 1,10 & 1,08 & 0,8708 & 1286,1 & 0,85 \\
\hline & 4 & 1,53 & 1,35 & 1,34 & 1,4492 & 1131,7 & 0,92 \\
\hline & 5 & 1,63 & 1,44 & 1,32 & 1,6223 & 1189,7 & 0,89 \\
\hline & 6 & 1,44 & 1,24 & 1,22 & 1,1406 & 1297,5 & 0,90 \\
\hline & 7 & 1,50 & 1,23 & 1,22 & 1,1786 & 1086,1 & 0,87 \\
\hline & 8 & 1,34 & 1,14 & 1,04 & 0,8318 & 1322,4 & 0,87 \\
\hline & 9 & 1,62 & 1,31 & 1,30 & 1,4445 & 1183,8 & 0,87 \\
\hline & 10 & 1,53 & 1,35 & 1,32 & 1,4276 & 1197,8 & 0,91 \\
\hline & Média & 1,50 & 1,29 & 1,25 & 1,3 & 1220 & 0,89 \\
\hline & D.P.* & 0,09 & 0,11 & 0,11 & 0,3 & 77 & 0,03 \\
\hline \multirow[t]{12}{*}{46,9} & 1 & 1,44 & 1,18 & 1,03 & 0,91639 & 1069,4 & 0,84 \\
\hline & 2 & 1,49 & 1,10 & 1,04 & 0,88418 & 1119,7 & 0,80 \\
\hline & 3 & 1,44 & 1,13 & 1,02 & 0,86602 & 1085,4 & 0,82 \\
\hline & 4 & 1,40 & 1,07 & 0,99 & 0,77651 & 1171,9 & 0,81 \\
\hline & 5 & 1,40 & 1,14 & 1,07 & 0,89097 & 1066,3 & 0,86 \\
\hline & 6 & 1,45 & 1,27 & 1,04 & 0,99451 & 995,5 & 0,86 \\
\hline & 7 & 1,39 & 1,16 & 0,96 & 0,79990 & 1037,6 & 0,83 \\
\hline & 8 & 1,39 & 1,09 & 0,98 & 0,77744 & 990,4 & 0,82 \\
\hline & 9 & 1,49 & 1,10 & 1,06 & 0,90662 & 1158,2 & 0,81 \\
\hline & 10 & 1,43 & 1,00 & 0,95 & 0,70509 & 1134,6 & 0,77 \\
\hline & Média & 1,43 & 1,12 & 1,01 & 0,85 & 1083 & 0,82 \\
\hline & D.P.* & 0,04 & 0,07 & 0,04 & 0,09 & 64 & 0,03 \\
\hline \multirow[t]{12}{*}{18,7} & 1 & 1,39 & 1,10 & 0,970 & 0,77304 & 1024,8 & 0,79 \\
\hline & 2 & 1,50 & 1,08 & 0,945 & 0,79787 & 976,1 & 0,81 \\
\hline & 3 & 1,27 & 1,03 & 0,920 & 0,62707 & 951,4 & 0,82 \\
\hline & 4 & 1,30 & 1,04 & 0,935 & 0,66189 & 1001,6 & 0,85 \\
\hline & 5 & 1,24 & 1,03 & 0,900 & 0,59944 & 716,2 & 0,83 \\
\hline & 6 & 1,44 & 1,02 & 0,895 & 0,68831 & 1047,3 & 0,74 \\
\hline & 7 & 1,29 & 1,00 & 0,925 & 0,62478 & 887,6 & 0,79 \\
\hline & 8 & 1,33 & 1,02 & 0,915 & 0,64749 & 906,3 & 0,84 \\
\hline & 9 & 1,40 & 1,05 & 0,890 & 0,68258 & 1030,4 & 0,78 \\
\hline & 10 & 1,32 & 1,05 & 0,920 & 0,66765 & 1000,1 & 0,80 \\
\hline & Média & 1,35 & 1,04 & 0,92 & 0,68 & 954 & 0,81 \\
\hline & DP* & 0,08 & 0,03 & 0,02 & 0,06 & 99 & 0,03 \\
\hline
\end{tabular}

* D.P. - Desvio Padrão; Dens. - densidade; Esf. - esfericidade; TU - Teor de Umidade de viscosidade do ar escoando no duto foram considerados iguais a $1,2050 \mathrm{~kg} \mathrm{~m}^{-3} \mathrm{e} 1,8135 \times 10^{-5} \mathrm{~Pa} . \mathrm{s}$, respectivamente.

Para a análise em que se empregou a técnica de elementos finitos, o sistema (Figura 2) foi dividido em 634 elementos, resultando em 693 nós. O sistema discretizado em elementos pode ser visto na Figura 4: (A) visão global e (B) visão ampliada da região do corpo sólido (fruto de café).

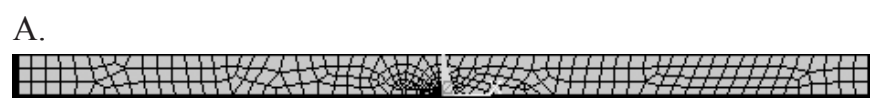

B.

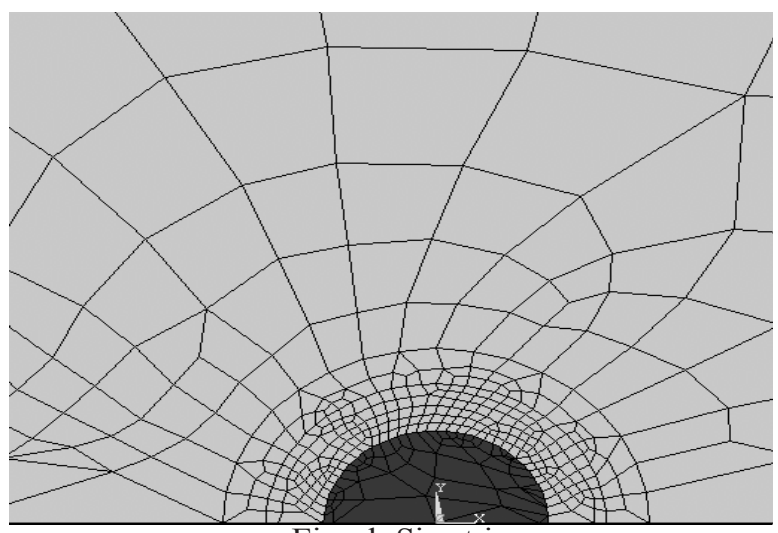

Eixo de Simetria

C.

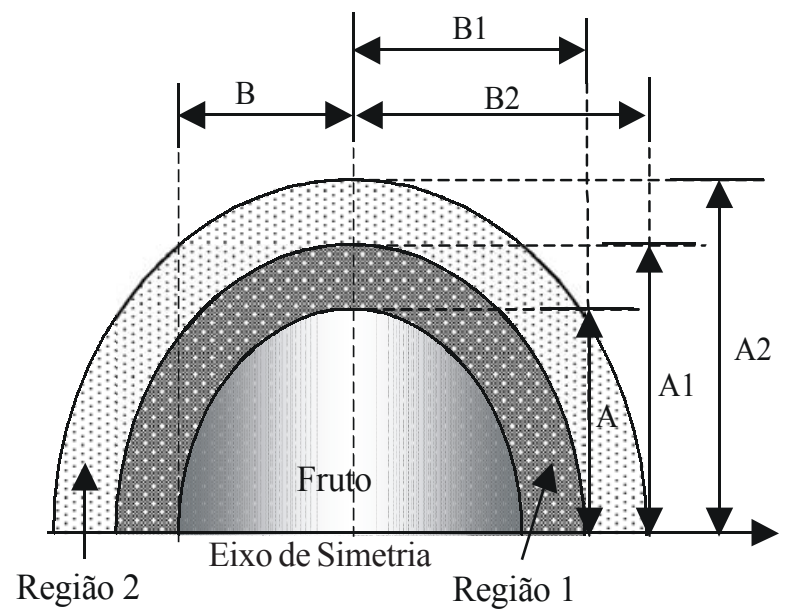

Figura 4. Discretização do sistema em elementos. (A) Visão global da discretização do sistema em elementos (B) Visão ampliada da discretização na região sólida (C) Regiões em torno do fruto

Sendo o número de elementos disponíveis para a discretização do sistema (versão 5.3 do ANSYS) limitado, construíram-se duas regiões contornando o fruto, como mostrado na Figura 4C, para se obter elementos de tamanhos menores nos locais de maiores gradientes de velocidade e de pressão. Os valores das dimensões A, A1, A2, B, B1 e B2 usados para cada fruto de café, são apresentados na Tabela 4 . No caso de fruto esférico, as dimensões A e B foram iguais ao raio da esfera equivalente (RE) e as duas regiões foram círculos de raios $\mathrm{Ra} 1=\mathrm{A} 1=\mathrm{B} 1$ e $\mathrm{Ra} 2=\mathrm{A} 2=\mathrm{B} 2$. 
Tabela 3. Velocidades terminais experimentais dos frutos de café, a diferentes teores de umidade, com respectivos desvios padrão (D.P.); peso real dos frutos; forças de arrasto simuladas para frutos esféricos e elípticos, com os respectivos erros relativos e viscosidade artificial (VA) usada nas simulações

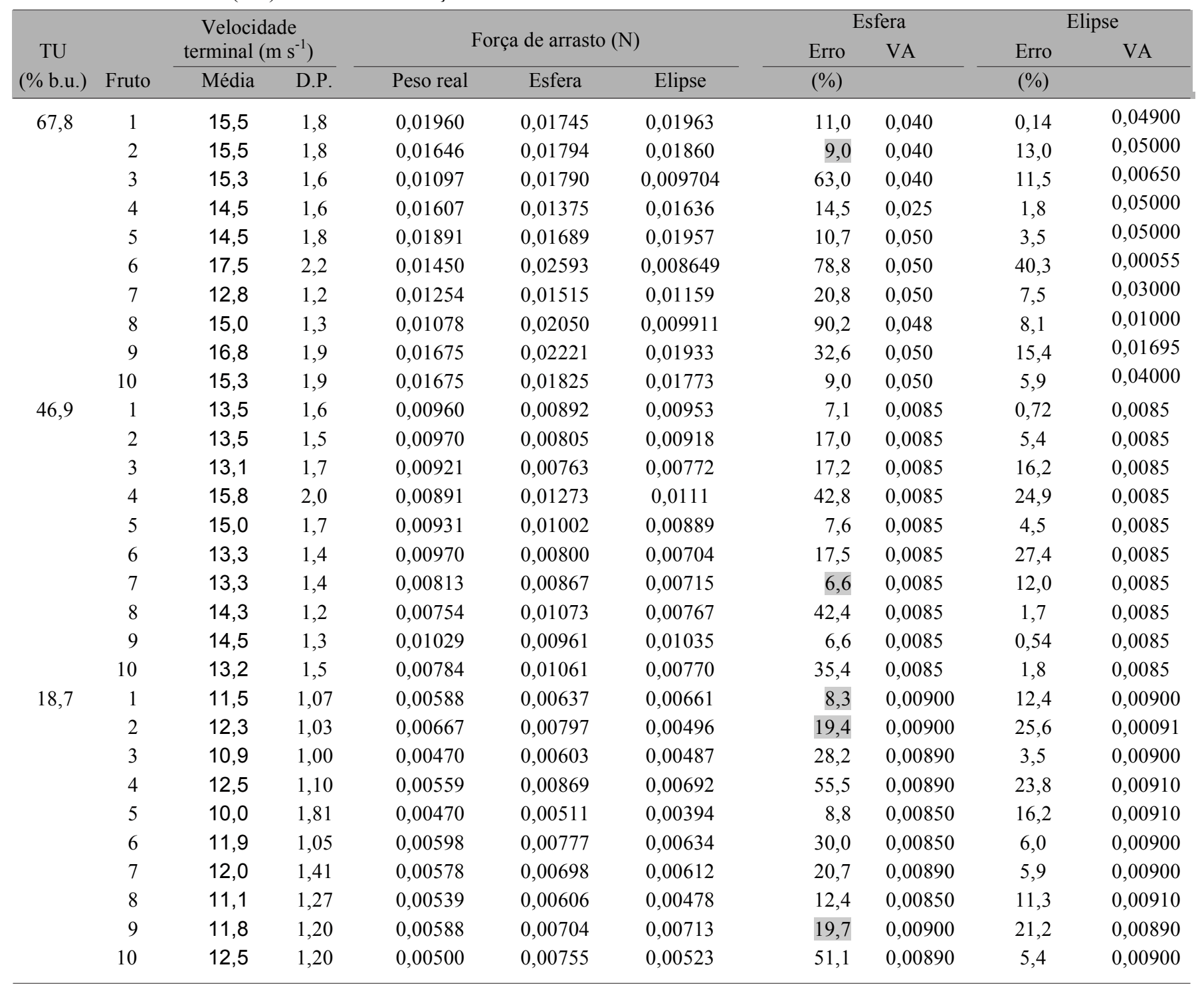

Neste trabalho, a introdução de uma viscosidade artificial (VA) para a execução do programa foi necessária para se obter convergência na solução. Os valores desta grandeza diferem para os frutos simulados e são mostrados na Tabela 3.

É interessante notar, na Tabela 3 que, em alguns casos, a consideração esférica para os frutos (células sombreadas), resultou em melhores resultados que a elíptica; entretanto, na maioria dos casos a consideração de uma forma elíptica para o produto produziu erros menores. Observa-se que os erros envolvidos nos valores das forças de arrasto para frutos com teores de umidade de 67,$8 ; 46,9$ e $18,7 \%$ b.u. variaram, respectivamente, de: (a) 9,0 a 90,2\%; 6,6 a 42,8\% e 8,3 a 55,5\%, para o fruto com forma esférica e (b) 0,14 a 40,3\%; 0,54 a 27,4\% e 3,5 a 25,6\%, para o fruto com forma elíptica. Em média, os erros envolvidos na estimativa da força de arrasto pela técnica de elementos finitos, para frutos considerados esfera e elipse foram, respectivamente, iguais a $34,0 \pm 31,4 \%$ e $10,711,5 \%$, para frutos a $67,8 \%$ b.u.; $20,014,8 \%$ e $9,510,2 \%$, para frutos a $46,9 \%$ b.u.; e $25,416,4 \%$ e $13,18,2 \%$, para frutos a $18,7 \%$ b.u.

\section{Forças de arrasto simuladas}

Os valores simulados das forças de arrasto usando-se a técnica de elementos finitos para frutos de café considerados esferas e elipses, são apresentados na Tabela 3. Os erros relativos envolvidos nos valores dessas forças encontram-se, também, na referida tabela. No cálculo desses erros considerouse que o peso do fruto, determinado experimentalmente (Tabela $3)$, é o valor mais correto da força de arrasto.

Um dos problemas mais comuns encontrados na solução de problemas envolvendo escoamento de um fluido, quando se usa o ANSYS, é a divergência ou oscilação da pressão e/ou campo de velocidade. Uma das maneiras de se reduzir o impasse é a introdução de uma viscosidade artificial visando suavizar a solução para a velocidade em regiões com grandes gradientes. No caso de fluidos incompressíveis, a introdução desta viscosidade não altera a solução final. Na prática, o valor da viscosidade artificial deve ser menor que 1000 vezes a viscosidade efetiva para problemas incompressíveis turbulentos (ANSYS Theory Manual, 1996). 
Tabela 4. Dimensões características $\left(10^{-3} \mathrm{~m}\right)$ das regiões usadas para contornar os frutos*

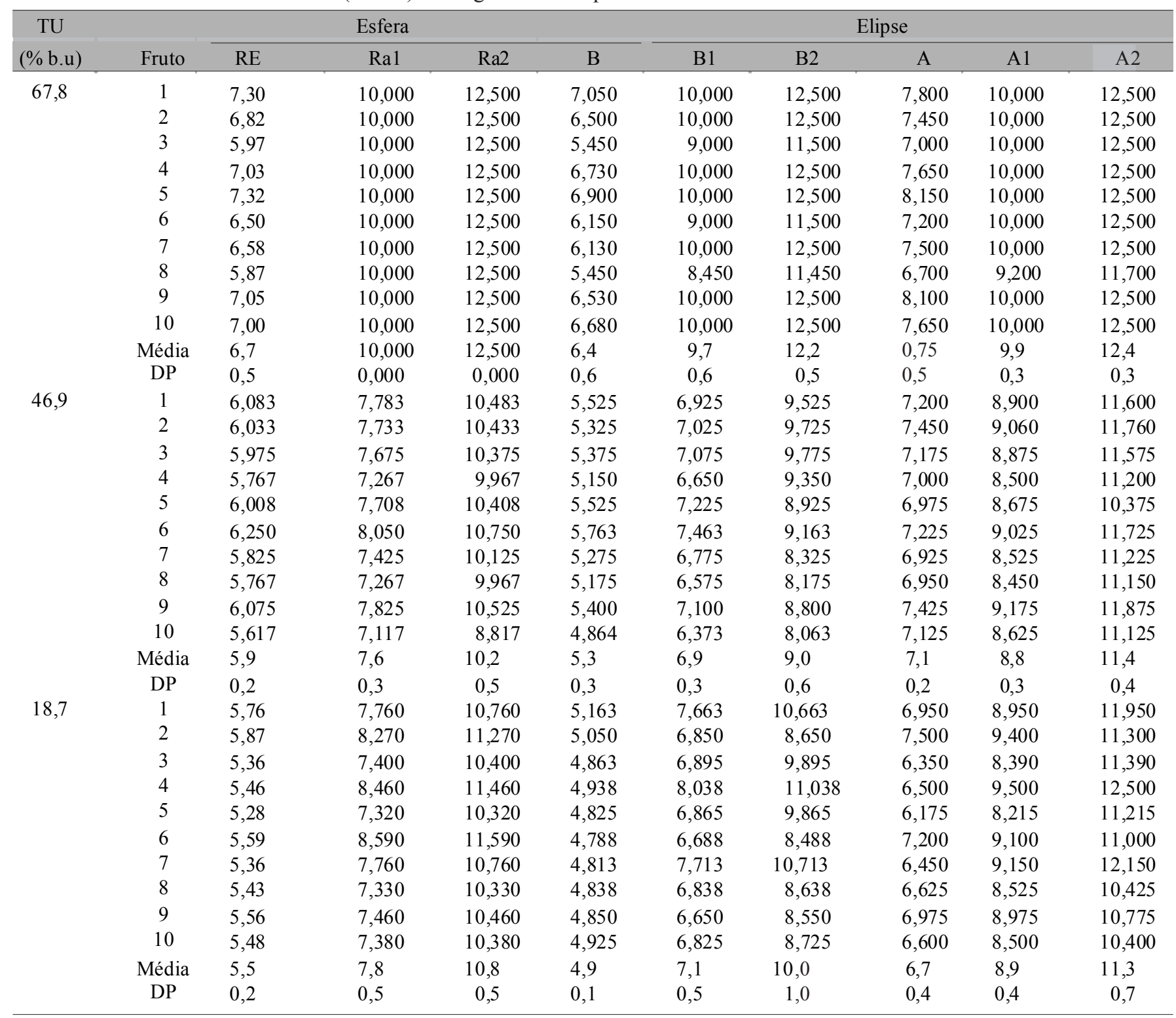

* TU - Teor de umidade; DP - Desvio padrão; Para RE, Ra1, Ra2, B, B1, B2, A1 e A2 ver Figura 4C.

Em termos de média em relação aos dez frutos, os valores da força de arrasto obtidos experimentalmente e por simulação, considerando-se o fruto esférico e elíptico foram, respectivamente, iguais a $0,015 \pm 0,003 ; 0,0190,004$ e $0,0150,005$ $\mathrm{N}$, para frutos a $67,8 \%$ b.u.; $0,0090,001 ; 0,0090,002$ e $0,0090,001$ $\mathrm{N}$, para frutos a $46,9 \%$ b.u. e $0,00560,0006 ; 0,0070,001$ e $0,0060,001 \mathrm{~N}$, para frutos a $18,7 \%$ b.u.

Em termos de média em relação aos dez frutos, os valores da força de arrasto obtidos experimentalmente e por simulação, considerando-se o fruto esférico e elíptico foram, respectivamente, iguais a $0,015 \pm 0,003 ; 0,0190,004$ e $0,0150,005 \mathrm{~N}$, para frutos a $67,8 \%$ b.u.; $0,0090,001 ; 0,0090,002$ e $0,0090,001 \mathrm{~N}$, para frutos a $46,9 \%$ b.u. e $0,00560,0006 ; 0,0070,001$ e $0,0060,001 \mathrm{~N}$, para frutos a $18,7 \%$ b.u.

Dos parâmetros que podem ter influenciado nos erros envolvidos nos valores simulados das forças de arrasto, citamse: (a) utilização de formas geométricas inadequadas para modelar o fruto de café; (b) considerações inadequadas na formulação do problema (forças cisalhantes e rugosidade na superfície do fruto desprezadas) e (c) incertezas nos valores experimentais da velocidade terminal (VT). Em média, as esfericidades dos frutos são iguais ou maiores que $85 ; 77$ e $74 \%$ para frutos com teores de umidade de 67,$8 ; 46,9$ e $18,7 \%$ b.u., respectivamente, e seus valores parecem não ter uma relação direta com os erros nos valores simulados das forças de arrasto de frutos considerados esféricos.

Acredita-se que as incertezas nos valores experimentais da velocidade terminal foram um dos parâmetros que mais influenciaram nos valores da força de arrasto. Essas incertezas se devem, principalmente, à rotação do fruto durante sua flutuação e, também, a desvios no posicionamento do sensor de velocidade na seção transversal do duto.

Testes de comparação de médias (Newman-Keuls, a um nível de significância de 5\%) mostraram que os valores simulados para força de arrasto dos frutos a 18,7 e $46,9 \%$ b.u., considerados elípticos e esféricos, não diferem dos pesos do produto determinados experimentalmente; entretanto, para o produto a $67,8 \%$ b.u., apenas a consideração elíptica para o fruto 
proporcionou forças de arrasto que não diferiram estatisticamente dos pesos experimentais do produto.

\section{Velocidades terminais simuladas}

Os valores das velocidades terminais provenientes das otimizações com os respectivos erros relativos, considerandose o valor experimental da velocidade terminal do produto como o mais correto, são apresentados na Tabela 5, para frutos de café, tidos como esferas e elipses, a diferentes teores de umidade.

Tabela 5. Valores das velocidades terminais ( Vt, $\mathrm{m} \mathrm{s}^{-1}$ ) provenientes das otimizações, com os respectivos erros relativos (Er, \%), ao se considerar os frutos a diferentes teores de umidade (TU, \% b.u.) como esferas e elipses

\begin{tabular}{ccccccccccc}
\hline & \multicolumn{10}{c}{ Fruto } \\
\cline { 2 - 5 } & 1 & 2 & 3 & 4 & 5 & 6 & 7 & 8 & 9 & 10 \\
\hline \multicolumn{10}{c}{ Elipse }
\end{tabular}

\begin{tabular}{|c|c|c|c|c|c|c|c|c|c|c|}
\hline 67,8 & $\mathrm{Vt}$ & 15,5 & 14,5 & 16,5 & 14,4 & 14,3 & 19,8 & 13,4 & $15,7 \quad 15,5$ & 14,8 \\
\hline & Er & 0,56 & 6,3 & 8,4 & 0,44 & 1,4 & 13,1 & 5,0 & 4,9 & 3,0 \\
\hline 46,9 & $\mathrm{Vt}$ & 13,6 & 13,9 & 14,5 & 14 & 15,4 & 16 & 14,3 & $14,2 \quad 14,5$ & 13,3 \\
\hline & Er & 0,74 & 3,0 & 3,7 & 7,7 & 2,7 & 20,8 & 7,9 & $0,350,33$ & 0,76 \\
\hline 18,7 & $\mathrm{Vt}$ & 10,8 & 14,5 & 10,7 & 11,1 & 11,1 & 11,5 & 11,6 & $11,9 \quad 10,6$ & 12,1 \\
\hline & $\mathrm{Er}$ & 6,1 & 17,9 & 1,8 & 11,2 & 11,0 & 3,4 & 3,3 & $7,2 \quad 10,2$ & 3,2 \\
\hline
\end{tabular}

\begin{tabular}{rrrrrrrrrrrr}
67,8 & $\mathrm{Vt}$ & 16,6 & 15,2 & 10,9 & 15,4 & 15,6 & 12,3 & 11,9 & 10,9 & 14,5 & 14,0 \\
& $\mathrm{Er}$ & 7,3 & 1,9 & 28,2 & 6,2 & 7,8 & 29,7 & 6,9 & 27,1 & 13,2 & 7,9 \\
46,9 & $\mathrm{Vt}$ & 14,0 & 14,8 & 14,5 & 12,8 & 14,4 & 14,9 & 12,8 & 11,6 & 15,1 & 11,0 \\
& $\mathrm{Er}$ & 1,0 & 3,2 & 10,6 & 11,3 & 2,6 & 20,5 & 7,9 & 0,53 & 0,33 & 1,0 \\
18,7 & $\mathrm{Vt}$ & 11,0 & 11,1 & 9,4 & 9,6 & 9,5 & 10,2 & 10,8 & 10,3 & 10,6 & 9,5 \\
& $\mathrm{Er}$ & 4,5 & 9,9 & 13,3 & 23,2 & 5,0 & 14,6 & 10,4 & 6,9 & 10,1 & 24,3 \\
\hline
\end{tabular}

É interessante notar, na Tabela 5, que os valores das velocidades terminais otimizadas dos frutos considerados esferas são, na maioria das vezes, menores que os obtidos experimentalmente para as velocidades terminais do produto. Os valores simulados para as velocidades terminais de 100, 50 e $70 \%$ dos frutos a 18,7; 46,9 e 67,8\% b.u., respectivamente, tendem a subestimar os valores das velocidades obtidas experimentalmente.

Em termos de média para os dez frutos, as velocidades determinadas experimentalmente e por simulação para frutos elípticos e esféricos foram, respectivamente iguais a: (a) 15,2 \pm 1,3; 15,51,8 e 13,72,1 $\mathrm{m} \mathrm{s}^{-1}$ para frutos a 67,8\% b.u.; (b) 13,90,9; $14,30,81$ e $13,71,4 \mathrm{~m} \mathrm{~s}^{-1}$ para o produto a $46,9 \%$ b.u. e (c) $11,70,8$; $11,61,1$ e $10,20,66 \mathrm{~m} \mathrm{~s}^{-1}$ para frutos a $18,7 \%$ b.u. Os menores erros relativos médios (para os dez frutos) aconteceram quando o fruto foi considerado elíptico. Os maiores erros relativos médios envolvidos nas simulações das velocidades terminais de frutos, nos três teores de umidade, considerados elípticos e esféricos, foram iguais a 7,5 e 13,6\%, respectivamente.

Testes de comparação de médias (Newman-Keuls, a um nível de significância de 5\%), aplicados aos dados experimentais, mostraram que os valores simulados de velocidade terminal para o produto elíptico a 18,7 e $67,8 \%$ b.u., não diferem daqueles determinados de forma experimental; entretanto, estes valores são diferentes daqueles simulados ao se considerar o fruto como uma esfera. Quando os frutos se encontravam a 46,9\% b.u., não se detectou diferença significativa entre os valores das velocidades terminais obtidas experimentalmente nem por simulações, ao se considerar os frutos elípticos e esféricos.

Portanto, valores de velocidade terminal de frutos de café a diferentes teores de umidade podem ser obtidos, por meio de simulação, conhecendo-se apenas o peso e as dimensões principais do produto.

\section{CONCLUSÕES}

1. A técnica dos elementos finitos mostrou-se ferramenta eficiente para simular forças de arrasto em frutos de café submetidos a um fluxo de ar.

2. Os valores de força de arrasto para o produto considerado com forma elíptica não diferiram estatisticamente dos pesos dos frutos determinados de maneira experimental.

3. A velocidade terminal de frutos de café pode ser obtida através de simulações usando-se a técnica dos elementos finitos; para tal, são necessários apenas dois parâmetros: o peso do produto e suas dimensões principais.

4. Independente do teor de umidade dos frutos, os valores simulados para a velocidade terminal e para força de arrasto no produto considerado elíptico são estatisticamente iguais àqueles determinados experimentalmente.

5. Os erros relativos médios (para os dez frutos) envolvidos no valores simulados para a velocidade terminal do produto em cada um dos teores de umidade, não foram superiores a 7,5 e $13,6 \%$ para o produto considerado elíptico e esférico, respectivamente.

\section{LITERATURA CITADA}

Allen, C.A.W.; Watts, K.C. Properties of cowpeas (var Minica Beans). Journal of Agricultural Engineering Research, Silsoe, v.68, n.2, p.159-167. 1997.

ANSYS Theory Reference. ANSYS Inc., Houston, PA, 1996.

Arsenijevic, Z.Lj.; Grbavcic, Z.B.; Garic-Grulovic, R.V.; Zdanski, F.K. Determination of non-spherical particle terminal velocity using particulate expansion data. Powder Technology, Amsterdam, v.103, n.3, p.265-273. 1999.

Carman, K. Some physical properties of lentil seeds. Journal of Agricultural Engineering Research, Silsoe, v.63, n.2, p. 87-92. 1996.

Chhabra, R.P.; Agarwal, L.; Sinha, N.K. Drag on non-spherical particles: an evaluation of available methods. Powder Technology, Elsevier, v.101, n.3, p. 288-295. 1999.

Dong, Z.; Gau, S.; Fryrear, D.W. Drag coefficients, roughness length and zero-plane displacement height as disturbed by artificial standing vegetation. Journal of Arid Environments, San Diego, v.49, n.3, p.485-505. 2001.

Fox, R.W.; McDonald, A.T. Introdução à mecânica dos fluidos. 4.ed. Rio de Janeiro: Livros Técnicos e Científicos Editora S.A., 1998. 662p.

Giles, R.V. Mecânica dos fluidos e hidráulica. 2.ed. São Paulo: McGraw-Hill do Brasil Ltda, 1980. 401p. 
Gupta, R.K.; Das, S.K. Physical properties of sunflower seeds. Journal of Agricultural Engineering Research, Silsoe, v.66, n.1, p.1-8. 1997.

Moaveni, S. Finite element analysis. Theory and Application with ANSYS. 1.ed. Upper Saddle River: Prentice-Hall, 1999. 527p.

Moreira, S.M.C.; Chaves, M.A.; Oliveira, L.M. Comparação da eficiência de líquidos na determinação da massa específica aparente de grãos agrícolas. Revista Brasileira de Armazenamento, Viçosa, v.9, n.1e2, p.22-24, 1984/85.

Nguyen, A.V.; Stechemesser, H.; Zobel, G.; Schulze, H.J. An improved formula for terminal velocity of rigid spheres. International Journal of Mineral Processing, Amsterdam, v.50, n.1-2, p.53-61. 1997.
Nimkar, P.M.; Chattopadhyay, P.K. Some physical properties of green gram. Journal of Agricultural Engineering Research, Silsoe, v.80, n.2, p.183-189. 2001.

Singh, K.K.; Goswami, T.K. Physical properties of cumin seed. Journal of Agricultural Engineering Research, Silsoe, v.64, n.2, p.93-98. 1996.

Suthar, S.H.; Das, S.K. Some physical properties of karingda [Citrullus lanatus (Thumb)] seeds. Journal of Agricultural Engineering Research, Silsoe, v.65, n.1, p.15-22. 1996.

Tabak, S.; Wolf, D. Aerodynamics properties of cottonseeds. Journal of Agricultural Engineering Research, Silsoe, v.70, n.3, p.257-265. 1998. 